\title{
Global changes of mRNA expression reveals an increased activity of the interferon-induced signal transducer and activator of transcription (STAT) pathway by repression of miR-221/222 in glioblastoma $\mathrm{U} 251$ cells
}

\author{
CHUNZHI ZHANG ${ }^{1,2,3^{*}}$, LEI HAN ${ }^{1,2,4^{*}}$, ANLING ZHANG ${ }^{1,2,4}$, WEIDONG YANG ${ }^{1}$, XUAN ZHOU $^{1,2}$, \\ PEIYU PU ${ }^{1,2}$, YUE DU ${ }^{2}$, HUAZONG ZENG ${ }^{5}$ and CHUNSHENG KANG $^{1,2,4}$
}

\author{
${ }^{1}$ Department of Neurosurgery, Tianjin Medical University General Hospital, Tianjin 300052; ${ }^{2}$ Laboratory of Neuro-oncology, \\ Tianjin Neurological Institute, Tianjin 300052; ${ }^{3}$ Department of Radiation Oncology, Tianjin HuanHu Hospital, \\ Tianjin 300060; ${ }^{4}$ Tianjin Key Laboratory of Injuries, Variations and Regeneration of Nervous System, \\ Tianjin 300052; ${ }^{5}$ Shanghai Sensichip Co. Ltd, Shanghai 200433, P.R. China
}

Received January 19, 2010; Accepted March 17, 2010

DOI: 10.3892/ijo_00000637

\begin{abstract}
MicroRNA (miR)-221 and miR-222 are frequently upregulated in various types of human malignancy including glioblastoma. Previous studies have identified some targets of miR-221 and miR-222, such as p27 and p57. Inter-relationship between miR-221 and miR-222 expression and global mRNA expression remains elusive. Here we knocked down miR-221 and miR-222 expression and found 158 differentially expressed genes with 2-fold changes in U251 glioma cells by microarray analysis. Using the KEGG pathway databases and BioCarta, we found that the IFN- $\alpha$ signaling pathway was the most significant pathway modulated by differentially expressed genes. STAT1 and STAT2 are core proteins in the IFN- $\alpha$ signaling pathway. By Western blotting and immunofluorescence, we found that STAT1 and STAT2 expression and phosphorylation were upregulated in U251 cells with knocked-down miR$221 / 222$. Furthermore, tyrosine phosphorylation of STAT1 and STAT2 was present in the nucleus after repression of miR-221/222 expression in U251 cells. These data indicate for the first time a mechanism involving STAT1/2 upregulation under the transcriptional control of INF- $\alpha$ signaling after knockdown of miR-221/222 cluster in U251 glioma cells.
\end{abstract}

\section{Introduction}

MiRNAs (miRs) are a class of highly conserved small noncoding single-stranded RNAs, of approximately $-22 \mathrm{nt}$, that

Correspondence to: Professor Chunsheng Kang, Laboratory of Neuro-Oncology, Tianjin Neurological Institute, Tianjin 300052, P.R. China

E-mail: kang97061@yahoo.com

*Contributed equally

Key words: glioma, miR-221, miR-222, STAT1, STAT2, INF- $\alpha$ play important regulatory roles at the post-transcriptional level (1). miRNA-mediated regulation of gene expression is considered to be one of the fundamental events at the post-transcriptional level (2-4). miRNAs participate in the determination of cell fate, pattern formation in embryonic development, and in the control of cell proliferation, cell differentiation and cell death (2-4). Recent research suggests the potential involvement of altered miRNAs in the pathogenesis of a wide range of human cancers $(2,3)$. Shortly after the cluster of human miRNAs was identified, miRNA cluster miR-15a-16, which includes miR-15a and miR-16-1, was identified located near $13 \mathrm{q} 14$, a region that is characterized by a high frequency of deletions in chronic lymphocytic lymphoma and several solid tumors (5). Another recent study indicated that expressions of miR-15a and miR-16-1 were inversely correlated with the Bcl-2 expression in chronic lymphatic leukemia, and that both of these miRNAs negatively regulate $\mathrm{Bcl}-2$ at the post-transcriptional level; thus potentiating the normal apoptotic response by targeting the anti-apoptotic gene Bcl-2 (6). This finding opens the door to the possibility of designing miRNA-based therapeutics. The best-characterized example of miRNA-based therapeutics is the miR-221/222 cluster. In a mouse subcutaneous tumor model for prostate carcinoma, the anti-miR-221/222 reduced tumor growth by increasing intratumoral p27 amount. The effect is long lasting, as it is detectable as long as 25 days after treatment (7). In general, previous studies have indicated that some miRNAs play an oncogenic role in different human cancers, but relatively little work has been done on the interrelationship between change of miRNAs expression and global changes of mRNA expression in the oncogenesis.

Gene expression analysis based on genome-wide microarray has been largely used to characterize human cancers. Initial microarray investigations support the belief that gliomas in general, and glioblastoma multiforme (GBM) in particular, are molecularly heterogeneous tumors, and that molecular approaches to classification and grading may have distinct diagnostic and prognostic advantages (8-14). This approach 
allowed the identification of genes involved in tumorigenesis (11-14). Furthermore, the discovery of gene expression signatures characteristic of distinctive clinic pathological features suggested that expression profiles could be used for molecular classification and evaluation of clinical prognosis of human gliomas (11-14). Microarray facilitates the comprehensive characterization of miRNA expression profiles in human cancers. Ciafre et al used microarray data to compare the global expression levels of 245 miRNAs in glioblastoma tissue to normal brain tissue, and found that the expressions of miR-221/222 were strongly upregulated in glioblastoma cells. They identified nine overexpressed miRNAs in glioblastoma cell lines, which including the miR-221/222 cluster. Thus, the overexpression of $\mathrm{miR}-221 / 222$ in glioblastoma cells might represent a molecular signature of glioblastoma tissues and cell lines (15). However, the gene expression profile reflecting the genomic changes after interruption of miR-221 and miR-222 expression in gliomas has received little attention.

In the present study, as indicated in Fig. 1, 2'-O-methyl (2'-OMe-) modified antisense oligonucleotides were used to knock down the expression of the miR-221/222 cluster in U251 cells. Global changes in gene expression were evaluated using a human 70-mer oligonucleotide microarray containing 35,000 well-characterized genes. The microarray analysis indicated that 145 genes were upregulated. Pathway analysis of the differentially expressed genes indicated significant changes in the activities of the IFN- $\alpha$ signaling pathway. We found evidence of differential regulation of transcription by eight transcription factors (TFs). We also demonstrated that STAT1 and STAT2 were the core factors affected by knockdown of miR-221/222. A mechanism involving STAT1/2 upregulation under the transcriptional control of INF- $\alpha$ signaling after the knockdown of miR-221/222 cluster in U251 glioma cells was proposed.

\section{Materials and methods}

Cell culture and transfection. Human glioma cell line U251, A172 and H4 were obtained from the Institute of Biochemistry and Cell Biology, Chinese Academy of Science. Cells were grown in Dulbecco's modified Eagle's medium (DMEM) (Gibco, USA) supplemented with $10 \%$ fetal bovine serum (Gibco), 2 mM glutamine (Sigma, USA), 100 units of penicillin/ml (Sigma) and $100 \mu \mathrm{g}$ of streptomycin/ml (Sigma). The cells were incubated at $37^{\circ} \mathrm{C}$ in $5 \% \mathrm{CO}_{2}$ and sub-cultured every 2-3 days. Total RNA was extracted from abovementioned cells by the TRIzol reagent (Invitrogen, Carlsbad, CA, USA) for miRNA profile examination. The miRNA microarray was obtained from CapitalBio Co. (Beijing, China).

The individual oligonucleotide probes were printed in triplicate on chemically-modified glass slides in a $21 \times 21$ spot configuration of each subarray for the miRNA microarray. All the oligonucleotide probes were printed in triplicate on one microarray, and each of the four subarrays contained 16 controls [Zip5, Zip13, Zip15, Zip21, Zip23, Zip25, Y2, Y3, U6, New-U2-R, tRNA-R, hsa-let-7a, hsa-let-7b, hsa-let-7c, 50\% DMSO (dimethyl sulphoxide) and Hex].

Total RNA (FirstChoice ${ }^{\circledR}$ Total RNA, Ambion, USA) from normal brain was used as a control. The miRNAs were

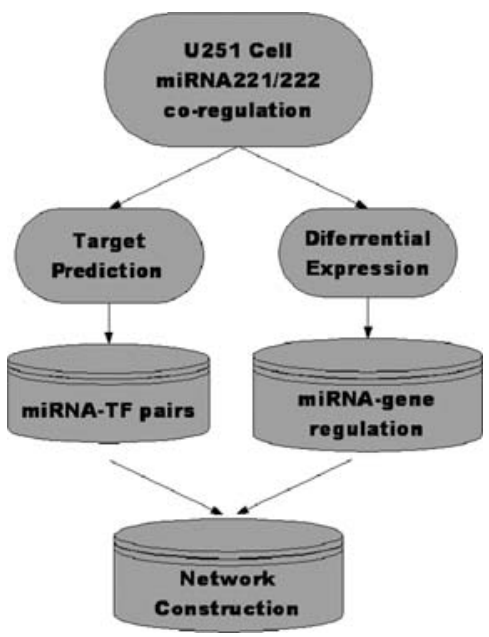

Figure 1. The miR-221 and miR-222 were found upregulated in four glioma cell lines by microarray. The knocked-down miR-221 and miR-222 in U251 cells were analyzed for the mRNA profile of changed genes. miRNA database was searched for the target genes of miR-221 and miR-222. By gene ontology (GO) and the KEGG pathway databases and BioCarta, a network of miR-221/222-TFs-STAT1/2-gene expression was constructed.

enriched from total RNA using a mirVana miRNA isolation kit (Ambion, USA) and labeled with mirVana array labeling kit (Ambion). Labeled miRNAs were used for hybridization on each miRNA microarray, containing 509 probes in triplicate, at $42^{\circ} \mathrm{C}$ overnight. A double-channel laser scanner (LuxScan 10K/A, CapitalBio) was used to scan the arrays and they were quantified by image analysis software (LuxScan 3.0, CapitalBio).

Transfection and RNA isolation. 2'-O-methyl (2'-OMe-) oligonucleotides were chemically synthesized by GenePharma Co., Ltd (Shanghai, China). 2'-O-Methyl oligos were composed entirely of 2'-O-methyl bases and had the following sequences: 2'OMe-miR-221 5'-GUCAACAUCAGUCUGAUA AGCUA-3' (AS-miR-221) and 2'OMe-miR-222 5'-GUC AACAUCAGUCUGAUAAGCUA-3' (AS-miR-222). The U251 cells were co-transfected with AS-miR221 and ASmiR-222 in equal amounts at a final concentration of $20 \mu \mathrm{M}$ using Lipofectamine 2000 (Invitrogen) according to manufacturer's protocol. Cells were cultured for $48 \mathrm{~h}$ until further study. Total RNA was isolated using the TRIzol reagent (Invitrogen) based on the manufacturer's protocol.

Microarray analysis. The 35,000-oligonucleotide microarray was constructed by CapitalBio Corp. using the Human Genome Oligo human-V4.0 (Operon), and comprises 35035 5-amino-modified 70-mer probes. In addition to internal controls provided by the Oligo Set vendor, twelve intergenic sequences from yeast were synthesized and used as external controls. After printing, the slides were baked for $1 \mathrm{~h}$ at $80^{\circ} \mathrm{C}$ and stored dry at room temperature until use. Prior to hybridization, the slides were dehydrated over $65^{\circ} \mathrm{C}$ water for $10 \mathrm{sec}$ and UV cross-linked at $250 \mathrm{~mJ} / \mathrm{cm}^{2}$ energy. The immobilized oligonucleotides were washed off with $0.5 \%$ SDS for $10 \mathrm{~min}$ at $42^{\circ} \mathrm{C}$ and SDS was removed by dipping the slides in anhydrous ethanol for $30 \mathrm{sec}$. An aliquot of $5 \mu \mathrm{g}$ 
Table I. miRNAs differentially expressed between human glioma cell lines and normal brain tissues by microarray SAM results (data in this table were normalized to ambion normal brain).

\begin{tabular}{|c|c|c|c|}
\hline miRNA & Chromosome location & Comparative expression of miRNAs & Potential targets \\
\hline \multicolumn{4}{|l|}{ Up-regulated } \\
\hline miR-21 & $17 \mathrm{q} 23.2$ & $7.035 \pm 2.409$ & PTEN, SEPT7 \\
\hline $\operatorname{miR}-15 b$ & $3 q 25.33$ & $5.433 \pm 1.700$ & CDC25A, EIF2C1 \\
\hline miR-106b & $7 q 22.1$ & $5.105 \pm 3.004$ & IGF2BP1, E2F1 \\
\hline $\operatorname{miR}-221$ & Xp11.3 & $3.496 \pm 0.948$ & TIMP3, LIFR \\
\hline $\operatorname{miR}-222$ & Xp11.3 & $3.404 \pm 1.079$ & ESR1, PAK1 \\
\hline $\operatorname{miR}-27 a$ & $19 \mathrm{p} 13.12$ & $3.327 \pm 0.953$ & MAP3K7IP3, SLC6A1 \\
\hline miR-23a & $19 \mathrm{p} 13.12$ & $3.183 \pm 0.365$ & ITGB8, PTEN \\
\hline $\operatorname{miR}-23 b$ & $9 q 23.31$ & $2.383 \pm 0.441$ & SEMA6D, TOP1 \\
\hline \multicolumn{4}{|c|}{ Down-regulated } \\
\hline miR-137 & $1 \mathrm{p} 21.3$ & $0.116 \pm 0.152$ & EIF3S1, SNF1LK \\
\hline $\operatorname{miR}-95$ & $4 \mathrm{p} 16.1$ & $0.114 \pm 0.138$ & NR4A2, OAZ2 \\
\hline $\operatorname{miR}-132$ & $17 \mathrm{p} 13.3$ & $0.093 \pm 0.075$ & GMFB, PAIP2 \\
\hline $\operatorname{miR}-128 b$ & $2 \mathrm{q} 21.3$ & $0.089 \pm 0.067$ & PDE3A \\
\hline $\operatorname{miR}-128 \mathrm{a}$ & $2 q 21.3$ & $0.084 \pm 0.061$ & EIF2S2, NF1 \\
\hline miR-342 & $14 q 32.2$ & $0.074 \pm 0.057$ & SMG7, SEPT3 \\
\hline $\operatorname{miR}-127$ & $14 q 32.31$ & $0.071 \pm 0.104$ & SEPT7, KIF3B \\
\hline $\operatorname{miR}-323$ & $14 q 32.31$ & $0.070 \pm 0.033$ & SEMA6D, IL11 \\
\hline $\operatorname{miR}-330$ & $19 q 13.32$ & $0.060 \pm 0.068$ & FGFR1, HOXD8 \\
\hline miR-1 & $20 q 13.33$ & $0.056 \pm 0.068$ & IGF1, TGIF2 \\
\hline $\operatorname{miR}-219$ & $6 \mathrm{p} 21.32$ & $0.037 \pm 0.055$ & NEUROD2 \\
\hline $\operatorname{miR}-126$ & $9 q 34.3$ & $0.025 \pm 0.009$ & IRS1 \\
\hline $\operatorname{miR}-329$ & $14 q 32.31$ & $0.021 \pm 0.011$ & PAM, TNRC6B \\
\hline miR-223 & $\mathrm{Xq} 12$ & $0.014 \pm 0.014$ & PDPK1, SLC37A3 \\
\hline $\operatorname{miR}-495$ & $14 q 32.31$ & $0.010 \pm 0.009$ & CDK6, BDNF \\
\hline $\operatorname{miR}-124 a$ & $20 q 13.33$ & $0.006 \pm 0.005$ & AMOTL1 \\
\hline miR-338 & $17 q 25.3$ & $0.006 \pm 0.003$ & FGFR2 \\
\hline miR-451 & $17 q 11.2$ & $0.004 \pm 0.003$ & CAB39, VAPA \\
\hline
\end{tabular}

of total RNA was used to produce fluorescent dye-labeled cDNA with Eberwine's linear RNA amplification method and a subsequent enzymatic reaction according to our previously published protocol (16). The labeled DNA was purified with PCR Clean-up NucleoSpin kits (Macherey Nagel, Germany), resuspended in Elution buffer, and quantified by UV spectrophotometry. Labeled control and test samples were quantitatively adjusted based on the efficiency of Cy-dye incorporation and mixed into $80^{\circ} \mathrm{C}$ hybridization solution (3X SSC, $0.2 \%$ SDS, $25 \%$ formamide and $5 \%$ Denhart's). DNA in hybridization solution was denatured at $95^{\circ} \mathrm{C}$ for 3 min prior loading on a microarray. Hybridization was performed under LifterSlip ${ }^{\mathrm{TM}}$ (Erie, USA), which allows for even dispersal of hybridization solutions between the microarray and coverslip. The hybridization chamber was placed on a 3-D tilting agitator BioMixer ${ }^{\mathrm{TM}}$ II (CapitalBio) to facilitate the microfluidic circulation under the coverslip. The array was hybridized at $42^{\circ} \mathrm{C}$ overnight and washed with two consecutive washing solutions $(0.2 \%$ $\mathrm{SDS}, 2 \mathrm{X} \mathrm{SSC}$ at $42^{\circ} \mathrm{C}$ for $5 \mathrm{~min}$, and $0.2 \% \mathrm{SSC}$ for $5 \mathrm{~min}$ at room temperature). After hybridization, microarrays were scanned with a confocal scanner LuxScan ${ }^{\mathrm{TM}} 10 \mathrm{KA}$
(CapitalBio), and the data from the obtained images were extracted with LuxScan 3.0 Software (CapitalBio). The raw data were normalized using a space and intensity-dependent Lowess program. The data from faint spots were removed, in which the intensity was lower than the average intensity plus 2 standard deviations of the negative controls on the array. The fidelity of the microarray had been previously evaluated $(17,18)$. Considering the very low false-positive ratio (data not shown), the ratio cut-off value of 2-fold was chosen for significant differential expression.

Functional group analysis. The microarray data were input into the Database for Annotation, Visualization and Integrated Discovery (DAVID, http://david.abcc.ncifcrf.gov/), which leveraged the Gene Ontology (GO) to identify the molecular function represented in the gene profile. In addition, we used the KEGG (Kyoto Encyclopedia of Genes and Genomes) database (http://www.genome.ad.jp/kegg/) and BioCarta (http://www.biocarta.com) to analyze the roles of these genes in the pathways. Literature mining was performed manually on the 145 genes and illustrated by graph-medusa (V 1.041, http://coot.embl.de/medusa). 
Table II. Functional classification of the 158 differentially expressed genes, by GO, and their over-representation.

\begin{tabular}{|c|c|c|c|c|c|}
\hline System & Term & Count & $\%$ & P-Value & Benjamini \\
\hline \multirow{16}{*}{$\begin{array}{l}\text { Biological } \\
\text { process }\end{array}$} & Protein kinase cascade & 7 & 4.5 & $8.4 \mathrm{E}-2$ & $1.0 \mathrm{E} 0$ \\
\hline & Induction of programmed cell death & 5 & 3.2 & $8.4 \mathrm{E}-2$ & $1.0 \mathrm{E} 0$ \\
\hline & Modification-dependent macromolecule catabolic process & 5 & 3.2 & $6.0 \mathrm{E}-2$ & $1.0 \mathrm{E} 0$ \\
\hline & Regulation of apoptosis & 9 & 5.7 & $5.7 \mathrm{E}-2$ & $1.0 \mathrm{E} 0$ \\
\hline & Regulation of progression through cell cycle & 9 & 5.7 & $5.1 \mathrm{E}-2$ & $1.0 \mathrm{E} 0$ \\
\hline & Protein catabolic process & 6 & 3.8 & $4.6 \mathrm{E}-2$ & $1.0 \mathrm{E} 0$ \\
\hline & Ectoderm development & 5 & 3.2 & $3.5 \mathrm{E}-2$ & $1.0 \mathrm{E} 0$ \\
\hline & Epidermis development & 5 & 3.2 & $2.8 \mathrm{E}-2$ & $1.0 \mathrm{E} 0$ \\
\hline & Regulation of programmed cell death & 10 & 6.4 & $2.5 \mathrm{E}-2$ & $1.0 \mathrm{E} 0$ \\
\hline & Cellular protein catabolic process & 6 & 3.8 & $1.7 \mathrm{E}-2$ & $1.0 \mathrm{E} 0$ \\
\hline & Cell death & 14 & 8.9 & $1.2 \mathrm{E}-2$ & $9.9 \mathrm{E}-1$ \\
\hline & Apoptosis & 14 & 8.9 & $7.3 \mathrm{E}-3$ & $9.8 \mathrm{E}-1$ \\
\hline & DNA packaging & 10 & 6.4 & $8.4 \mathrm{E}-4$ & $4.2 \mathrm{E}-1$ \\
\hline & Establishment and/or maintenance of chromatin architecture & 10 & 6.4 & $7.4 \mathrm{E}-4$ & 4.7E-1 \\
\hline & Potein-DNA complex assembly & 8 & 5.1 & $1.9 \mathrm{E}-4$ & $2.2 \mathrm{E}-1$ \\
\hline & Nucleosome assembly & 8 & 5.1 & $5.5 \mathrm{E}-6$ & $1.4 \mathrm{E}-2$ \\
\hline \multirow{7}{*}{$\begin{array}{l}\text { Cellular } \\
\text { component }\end{array}$} & Proteasome complex (sensu Eukaryota) & 3 & 1.9 & 7.9E-2 & $1.0 \mathrm{E} 0$ \\
\hline & Intermediate filament cytoskeleton & 5 & 3.2 & $7.2 \mathrm{E}-2$ & $1.0 \mathrm{E} 0$ \\
\hline & Intermediate filament & 5 & 3.2 & $7.2 \mathrm{E}-2$ & $1.0 \mathrm{E} 0$ \\
\hline & Chromosome & 10 & 6.4 & $5.5 \mathrm{E}-3$ & $6.1 \mathrm{E}-1$ \\
\hline & Chromosomal part & 10 & 6.4 & $1.8 \mathrm{E}-3$ & $3.4 \mathrm{E}-1$ \\
\hline & Chromatin & 10 & 6.4 & $4.0 \mathrm{E}-5$ & $1.4 \mathrm{E}-2$ \\
\hline & Nucleosome & 8 & 5.1 & $2.5 \mathrm{E}-6$ & $1.7 \mathrm{E}-3$ \\
\hline \multirow{4}{*}{$\begin{array}{l}\text { Molecular } \\
\text { function }\end{array}$} & GPI anchor binding & 4 & 2.5 & $6.9 \mathrm{E}-2$ & $1.0 \mathrm{E} 0$ \\
\hline & NAD+ADP-ribosyltransferase activity & 3 & 1.9 & $1.4 \mathrm{E}-2$ & $9.9 \mathrm{E}-1$ \\
\hline & Copper ion binding & 5 & 3.2 & $4.6 \mathrm{E}-3$ & $8.9 \mathrm{E}-1$ \\
\hline & Cadmium ion binding & 4 & 2.5 & $2.4 \mathrm{E}-5$ & $2.3 \mathrm{E}-2$ \\
\hline
\end{tabular}

Immunofluorescence analysis. Transfected cells were seeded on coverslips and fixed with $4 \%$ paraformaldehyde (PFA, Sigma), treated with $3 \% \mathrm{H}_{2} \mathrm{O}_{2}$ for 10 min and incubated with the PUMA or Bax antibodies (Santa Cruz Biotechnology, CA, USA) overnight at $4^{\circ} \mathrm{C}$. FITC- or TRITC-labeled secondary antibody was then added (1:200 dilution) for $2 \mathrm{~h}$ at $37^{\circ} \mathrm{C}$. After staining with DAPI dye for $5 \mathrm{~min}$, the cells were visualized on a FV-1000 laser scanning confocal microscope and analyzed by IPP5.1 software (Olympus, Japan).

Western blot analysis. Western blot analysis was used to characterize the expression of proteins after gene knockdown by co-transfection with AS-miR-221 and AS-miR-222 in U251 cells (19). Equal amounts $(20 \mu \mathrm{g})$ of protein extracts from each sample were resolved by SDS-PAGE on $8 \%$ gels. Proteins were probed with mouse anti-STAT1, STAT2 and phospho-STAT1, STAT2 monoclonal antibodies $(1: 1,000$ dilution, Santa Cruz Biotechnology), and B-actin (1:2,000 dilution, Santa Cruz Biotechnology) followed by incubation with a horseradish peroxidase-conjugated secondary antibody (1:2,500 dilution, Zhongshan, China). Proteins were visualized with a chemiluminescence detection system using the Super Signal substrate (Pierce, USA).
Statistical analysis. Data are expressed as means $\pm \mathrm{SE}$, and $\mathrm{P}<0.01$ is considered as statistically significant by ANOVA, $\chi^{2}$ test and SNK t-test.

\section{Results}

Knockdown of the miR-221/222 cluster in U251 cell line. To investigate whether miRNAs are differentially expressed in glioma cells versus normal brain tissue, we performed a microarray analysis using a chip able to examine the global expression levels of 469 miRNAs. The results showed that miR-15b, miR-106b, miR-221, miR-27a, miR-21, miR-222, miR-23b and miR-23a were upregulated in glioma cells (Table I). In particular, miR-221 and miR-222 were strongly upregulated in glioma cells (Fig. 2). This result was similar to the result of Ciafre et al (15).

Our study indicated a single miR-221 antagomiR did not affect cellular growth in U251 glioblastoma cells in vitro, indicating a functional overlap between miR-221 and miR222 in controlling cell survival (20). Noting this, we used an antisense method to co-suppress the expression of miR221/222 in U251 cells. We found that miR-221/222 were downregulated by co-transfecting U251 cells with AS-miR- 

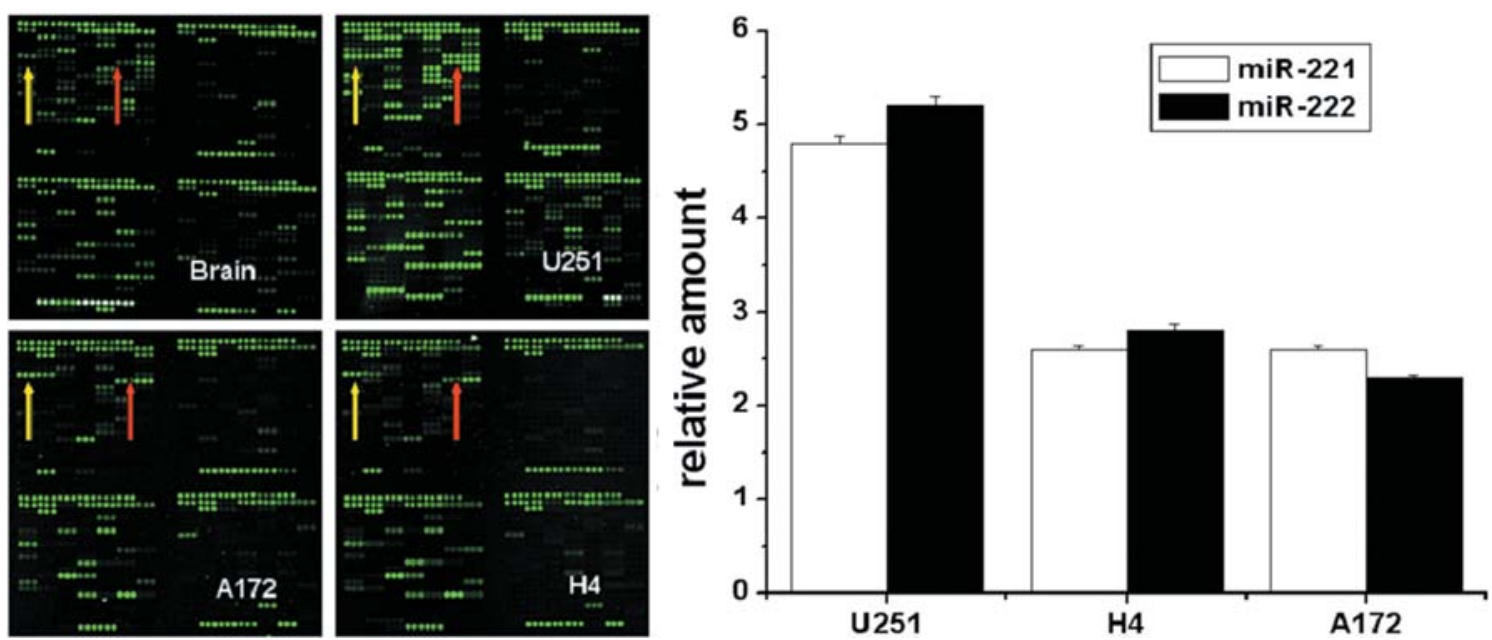

Figure 2. miRNA microarray analysized miRNAs prolife in glioma cell lines. The cluster analysis of miRNA microarray data showed miR-221 and miR-222 overexpression in four human glioma cell lines.

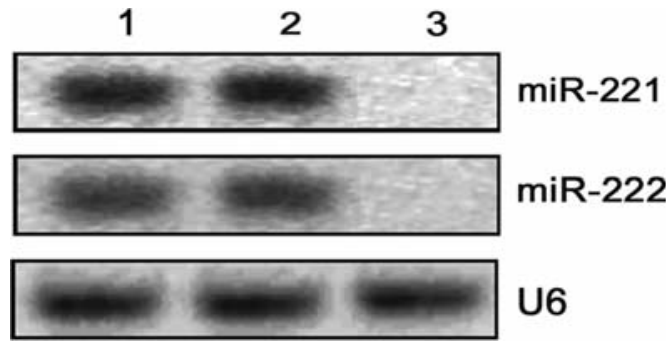

Figure 3. Northern blot analysis of miR-221 and miR-222 expression. Northern blot detection of miR-221/222 expression in U251 glioblastoma cells after co-suppression by antisense oligonucleotides (1, control; 2, scrambled miR; 3, As-miR-221/222).

221/222. Northern blotting using non-isotopic detection of miRNA with digoxigenin-labeled RNA probes showed that AS-miR-221/222 was effectively blocking miR-221/222 expression, while scramble transfection yielded no effect on miRNA expression (Fig. 3). All of the tested microRNAs were predominantly present in their mature form in U251 cells.

Microarray analysis of expression of INF- $\alpha$ pathway by repression of miR-221/222 in U251 glioma cells. Previously, Guo et al compared CapitalBio's long oligonucleotide (Operon 70-mer) microarray with Affymetrix's short oligonucleotide (25-mer) HG U133A 2.0 array (21). Employing the same batch of RNA extracted from HeLa and HEK293 cell lines, the correlation coefficient (R-value) between the two platforms was 0.787 , and the reverse fluorescence strategy in CapitalBio's platform was proved to give a high level of reproducibility between two independent experiments $(21,22)$. A large-scale microarray analysis was used to investigate the changes in mRNA expression profiles after repression of the miR-221/222 cluster in U251 glioma cells.

We identified 158 differentially expressed genes with 2 -fold changes after repression of the miR-221/222 cluster in U251 glioma cells. Among them, 145 were upregulated and 13 were downregulated. We took several approaches to identify the potential functional significance of the differentially expressed genes. We used gene ontology (GO) to classify each differentially expressed gene into functional categories and determined if any categories were overrepresented compared to all genes on the array. The most over-represented GO molecular function categories related to the protein kinase cascade and induction of programmed cell death (Table II). Other significant GO-defined categories included modification-dependent macromolecule catabolic processes, regulation of apoptosis, and regulation of progression through the cell cycle (Table II).

We also evaluated the pathways linking the differentially expressed genes. Using the KEGG pathway databases and BioCarta, we found that the antigen processing and presentation in KEGG and the IFN- $\alpha$ signaling pathway in KEGG and BioCarta were the most significant pathways modulated by the differentially expressed genes. The IFN- $\alpha$ signaling pathway contained 17 genes that were upregulated in miR221/222 knocked-down U251 cells compared to parental U251 cells (Table III).

We then determined whether the differentially expressed genes were the target genes of miR-221/222. Three databases (TargetScan, PicTar, miRanda) were used for miRNA target prediction. A total of 70 candidate genes were potential targets of the miR-221/222 cluster (Table IV). Intriguingly, no intersection was found between differentially expressed genes and the predicted target genes, suggesting an indirect regulation between the miR-221/222 cluster and mRNA expression, possibly via transcription factors (TFs).

We chose TRANSFAC ${ }^{\circledR} 7.0$ Public (http://www.generegulation.com/ pub/databases.html) as the transcription factor database to identify the TFs potentially regulated by the miR-221/222 cluster. Eight TFs were found to be regulated by the miR-221/222 cluster (Table V). Literature data mining was used to predict relationships between the genes, identifying 28 genes and 64 verified modulation nodes. Our results support the view that IRF-1, STAT1 and STAT2 regulate the balance between miR-221/222-TFs and TF-gene expression, determining the cell death or survival through the INF- $\alpha$ pathway in glioma U251 cells (Fig. 4). 
Table III. Upregulation of members of the IFN- $\alpha$ signaling pathway in miR-221/222 knocked-down U251 cells. ${ }^{a}$

\begin{tabular}{lrl}
\hline Name & WT $^{+} /$WT $^{-b}$ & \\
\hline CXCL10 & 7.5706 & Description of RefSeq $^{c}$ \\
GBP1 & 3.7516 & Small inducible cytokine B10 precursor (CXCL10) \\
IFI16 & 2.5931 & Interferon-induced guanylate-binding protein 1 \\
IFI27 & 10.5238 & $\gamma$-interferon-inducible protein Ifi-16 \\
IFI30 & 4.2318 & Interferon- $\alpha$-induced 11.5 kDa protein \\
IFI35 & 6.8096 & $\gamma$-interferon inducible lysosomal thiol reductase precursor \\
IFI44 & 9.8163 & Interferon-induced 35 kDa protein (IFP 35) \\
IFIT1 & 18.1824 & Interferon-induced protein 44 \\
IFIT2 & 21.1874 & Interferon-induced protein with tetratricopeptide repeats 1 \\
IFIT3 & 39.4750 & Interferon-induced protein with tetratricopeptide repeats 2 \\
IFIT5 & 4.2232 & Interferon-induced protein with tetratricopeptide repeats 3 \\
IFITM1 & 13.7500 & Interferon-induced protein with tetratricopeptide repeats 5 \\
IFITM2 & 8.8921 & Interferon-induced transmembrane protein 1 \\
IRF1 & 2.8741 & Interferon-induced transmembrane protein 2 \\
ISGF3G & 2.6395 & Interferon regulatory factor 1 \\
STAT1 & 5.5881 & Transcriptional regulator ISGF3 $\gamma$ subunit \\
STAT2 & 2.4067 & Signal transducer and activator of transcription 1 \\
\hline
\end{tabular}

${ }^{a}$ The genes whose expression was upregulated 2 -fold. ${ }^{b} W^{+}$, U251 cell; WT', U251 cells with miR-221/222 knockdown. ${ }^{\text {Reference }}$ sequence (RefSeq).

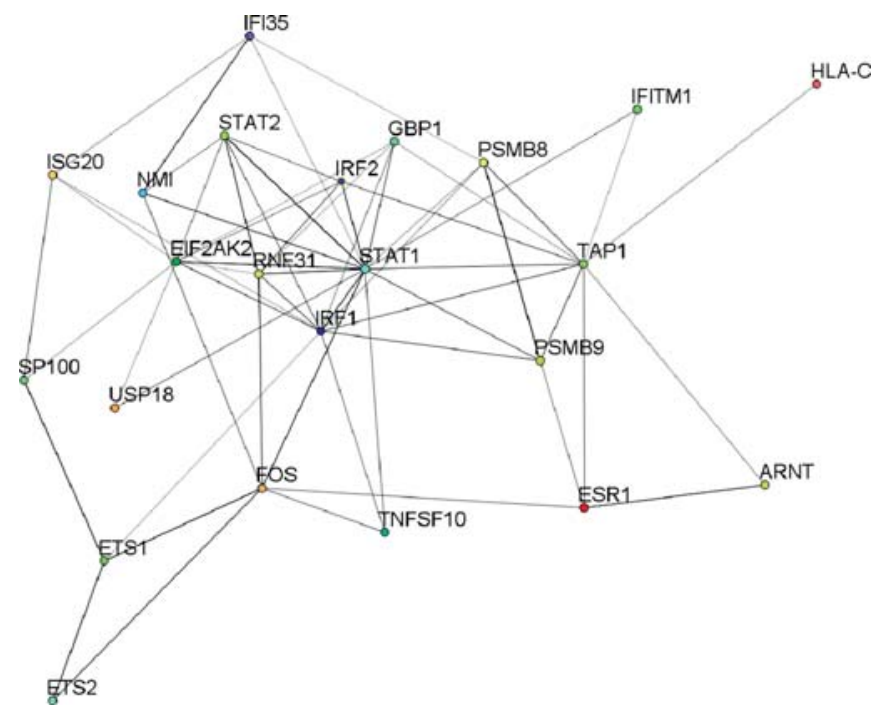

Figure 4. Constructed network of miR-221/222-TFs-STAT1/2-gene expression. Genes involved in TF-gene expression in glioma U251 cells after repression of the miR-221/222 cluster, determining the cell death or survival through the INF- $\alpha$ pathway. Genes associated with eight TFs (FOS, ESR1, ETS2, IRF2, ARNT, ETS1, HOXC1, FOXN2) were manually identified via literature mining. IRF-1, STAT1 and STAT2 regulate the balance between miR-221/222-TFs and TF-gene expression, determining the cell death or survival through the INF- $\alpha$ pathway in glioma U251 cells. The INF- $\alpha$ pathway regulates cell survival negatively, via processes occurring in multiple intracellular compartments. Regulatory relationships are denoted by line colors, including the 28 genes and 64 verified modulation nodes.

miR-221/222 modulate STAT1 and STAT2 tyrosine phosphorylation and nuclear translocation. Dephosphorylation and tyrosine phosphorylation of STAT1 and STAT2 were detected

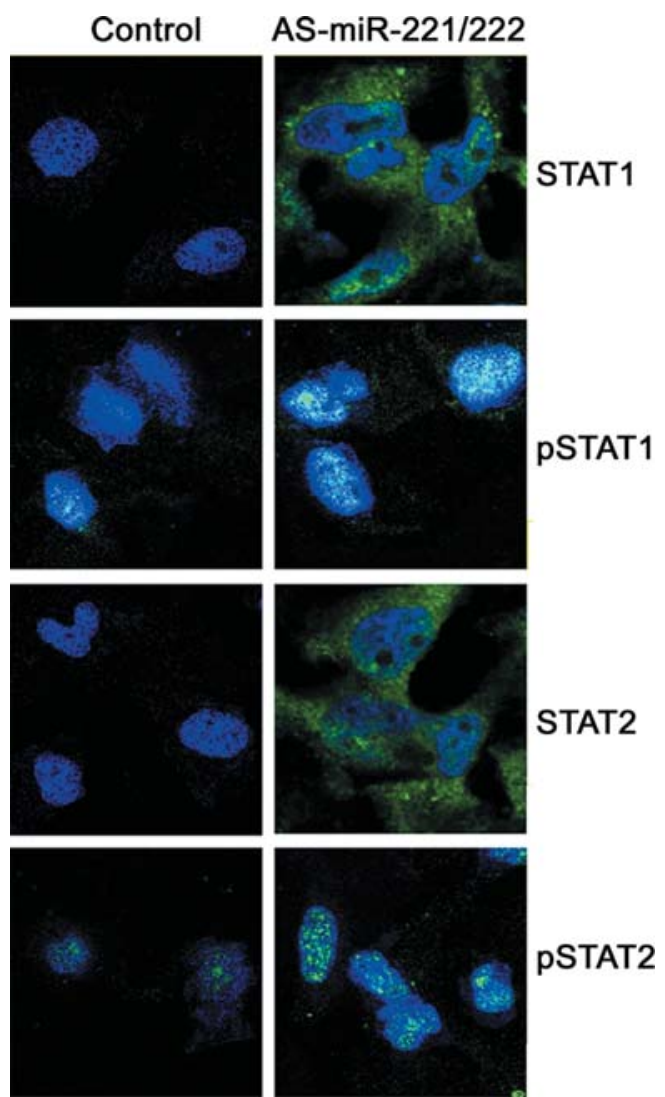

Figure 5. Immunofluorescence indicates dephosphorylation and phosphorylation of STAT1 and STAT2 expression and location. After co-suppression of the miR-221/222 cluster in U251 glioblastoma cells, dephosphorylation of STAT1 and STAT2 expression increased in the cytoplasm, tyrosine phosphorylation of STAT1 and STAT2 also increased in the nucleus. 
Table IV. Target prediction of miR-221/222 (TargetScan, PicTar, miRanda).

\begin{tabular}{|c|c|}
\hline Gene symbol & Gene name \\
\hline CDC2L6 & Cell division cycle 2-like 6 (cdk8-like) \\
\hline HOXC10 & Homeobox c10 \\
\hline ETS1 & v-ets erythroblastosis virus e26 oncogene homolog 1 (avian) \\
\hline KIAA 1267 & dkfzp727c091 protein \\
\hline RALA & v-ral simian leukemia viral oncogene homolog a (ras related) \\
\hline MYLIP & Myosin regulatory light chain interacting protein \\
\hline VAPB & Vamp (vesicle-associated membrane protein)-associated protein $\mathrm{b}$ and $\mathrm{c}$ \\
\hline ESR1 & Estrogen receptor 1 \\
\hline PAIP2 & Poly(a) binding protein interacting protein 2 \\
\hline RAB1A & rab1a, member of the ras oncogene family \\
\hline IGF2BP2 & Insulin-like growth factor 2 mRNA-binding protein 2 \\
\hline RBM24 & RNA-binding motif protein 24 \\
\hline SNCB & Synuclein, $\beta$ \\
\hline PPP3R1 & Protein phosphatase 3 (formerly $2 \mathrm{~b}$ ), regulatory subunit $b, 19 \mathrm{kda}, \alpha$ isoform (calcineurin $b$, type i) \\
\hline TRPS1 & Trichorhinophalangeal syndrome i \\
\hline HECTD2 & Hect domain containing 2 \\
\hline ADAM11 & Adam metallopeptidase domain 11 \\
\hline ARID1A & At rich interactive domain 1a (swi-like) \\
\hline PPARGC1A & Peroxisome proliferative-activated receptor, $\gamma$, coactivator $1, \alpha$ \\
\hline ETS2 & v-ets erythroblastosis virus e26 oncogene homolog 2 (avian) \\
\hline TMCC1 & Transmembrane and coiled-coil domain family 1 \\
\hline POGZ & Pogo transposable element with znf domain \\
\hline DNAJC14 & Cytokine-induced protein $29 \mathrm{kda}$ \\
\hline C12orf30 & Chromosome 12 open reading frame 30 \\
\hline TIMP3 & Timp metallopeptidase inhibitor 3 (sorsby fundus dystrophy, pseudoinflammatory) \\
\hline MAP3K10 & Mitogen-activated protein kinase kinase kinase 10 \\
\hline FOS & v-fos fbj murine osteosarcoma viral oncogene homolog \\
\hline PPP6C & Protein phosphatase 6 , catalytic subunit \\
\hline CDV3 & cdv3 homolog (mouse) \\
\hline FNDC3A & Fibronectin type iii domain containing $3 \mathrm{a}$ \\
\hline GNAI2 & Guanine nucleotide-binding protein (g protein), $\alpha$-inhibiting activity polypeptide 2 \\
\hline ZFPM2 & Zinc finger protein, multitype 2 \\
\hline VGLL4 & Vestigial like 4 (Drosophila) \\
\hline FAT2 & Fat tumor suppressor homolog 2 (Drosophila) \\
\hline ANKHD1 & Ankyrin repeat and kh domain containing 1 \\
\hline KIAA1598 & kiaa1598 \\
\hline NAP1L5 & Nucleosome assembly protein 1-like 5 \\
\hline PHF2 & phd finger protein 2 \\
\hline INSIG1 & Insulin-induced gene 1 \\
\hline PAIP1 & Poly(a) binding protein interacting protein 1 \\
\hline $\mathrm{BMF}$ & bcl2 modifying factor \\
\hline IRX5 & Iroquois homeobox protein 5 \\
\hline YWHAG & Tyrosine 3-monooxygenase/tryptophan 5-monooxygenase activation protein, $\gamma$ polypeptide \\
\hline HRB & hiv-1 rev binding protein \\
\hline PDCD10 & Programmed cell death 10 \\
\hline CDKN1C & Cyclin-dependent kinase inhibitor 1c (p57, kip2) \\
\hline HIPK1 & kiaa0630 protein \\
\hline CCDC64 & Coiled-coil domain containing 64 \\
\hline ASB7 & Hypothetical protein flj22551 \\
\hline RSBN1L & Round spermatid basic protein 1-like \\
\hline MAPK10 & Mitogen-activated protein kinase 10 \\
\hline IRF2 & Interferon regulatory factor 2 \\
\hline
\end{tabular}


Table IV. Continued.

\begin{tabular}{ll}
\hline Gene symbol & Gene name \\
\hline MESDC1 & Mesoderm development candidate 1 \\
NLK & Nemo-like kinase \\
ARF4 & adp-ribosylation factor 4 \\
RIMS3 & Regulating synaptic membrane exocytosis 3 \\
KHDRBS2 & kh domain containing, RNA binding, signal transduction associated 2 \\
PBX3 & Pre-b-cell leukemia transcription factor 3 \\
DMRT3 & Doublesex and mab-3 related transcription factor 3 \\
GARNL1 & kiaa0884 protein \\
DACH1 & Dachshund homolog 1 (Drosophila) \\
ANGPTL2 & Angiopoietin-like 2 \\
MSL2L1 & Male-specific lethal 2-like 1 (Drosophila) \\
TOX & Thymus high mobility group box protein tox \\
ARNT & Aryl hydrocarbon receptor nuclear translocator \\
ATXN1 & Ataxin 1 \\
INA & Internexin neuronal intermediate filament protein, $\alpha$ \\
SLC25A37 & Solute carrier family 25, member 37 \\
MIER3 & \\
ZEB2 & \\
\hline
\end{tabular}

Table V. miR-221and miR-222 co-regulated transcription factors.

\begin{tabular}{|c|c|}
\hline Gene symbol & Gene name \\
\hline FOS & $\begin{array}{l}\text { v-fos fbj murine osteosarcoma viral } \\
\text { oncogene homolog }\end{array}$ \\
\hline ESR1 & Estrogen receptor 1 \\
\hline ETS2 & $\begin{array}{l}\text { v-ets erythroblastosis virus e } 26 \\
\text { oncogene homolog } 2 \text { (avian) }\end{array}$ \\
\hline IRF2 & Interferon regulatory factor 2 \\
\hline ARNT & $\begin{array}{l}\text { Aryl hydrocarbon receptor } \\
\text { nuclear translocator }\end{array}$ \\
\hline ETS1 & $\begin{array}{l}\text { v-ets erythroblastosis virus e } 26 \\
\text { oncogene homolog } 1 \text { (avian) }\end{array}$ \\
\hline $\mathrm{HOXC1}$ & Homeobox protein $\mathrm{HOXC1}$ \\
\hline FOXN2 & Forkhead box N2 \\
\hline
\end{tabular}

using immunostaining and immunoblotting techniques. Fig. 5 shows that dephosphorylation of STAT1 and STAT2 occurs in the cytoplasm. Fig. 5 also shows that tyrosine phosphorylation of STAT1 and STAT2 was present in the nucleus after repression of miR-221/222 expression in U251 cells. This rapid onset in STAT1 and STAT2 immuno-reactivity after miR-221/222 repression probably represents increased protein affinity and/or accessibility to the antibody consequent to STAT1/STAT2 mobilization and dimerization. Finally, Western blotting confirmed these results (Fig. 6).

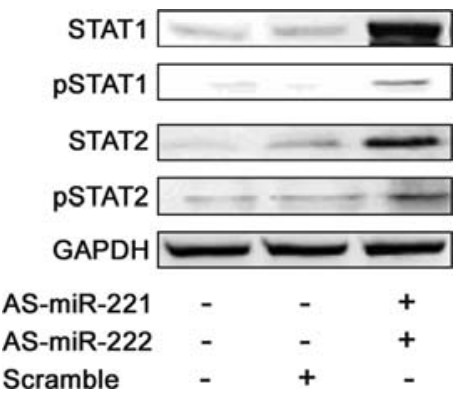

Figure 6. Western blot analysis of dephosphorylation and phosphorylation of STAT1 and STAT2 expression. After co-suppression of the miR-221/222 cluster in U251 glioblastoma cells, dephosphorylation of STAT1 and STAT2 expression increased, and tyrosine phosphorylation of STAT1 and STAT2 also increased.

Not unexpectedly, the expression of STAT1 and STAT2 and their activated form (phosphorylated STAT1 and STAT2) were greater after repression of the miR-221/222 cluster than that in parental U251 cells (Fig. 6).

Our results revealed that knockdown of miR-221/222 changed the mRNA expression profiles of genes that are not target genes of miR-221/222 in U251 cells. In the regulatory networks that are affected by knockdown of miR-221/222, the mRNA expression profiles of STAT1 and STAT2 are central to the effect. That is, knockdown of miR-221/222 upregulated STAT1 and STAT2 expression and their phosphorylation to change mRNA expression profiles.

\section{Discussion}

A growing number of papers have been published describing a link between several forms of human cancer and the 
expression of miRNAs (3). The majority of the studies identified targets of the miRNAs that were shown to be specifically modulated in tumors. Compared with the regulation of transcription, the study of the regulatory networks influenced by miRNA is only just beginning (23). In this study, we focused on miRNAs, miR-221/222, that are clustered at Xp11.3, which had been demonstrated to overexpress in the specific context of cancers $(2,15,24,25)$. Both miRNAs are clustered within a $1-\mathrm{kb}$ genomic interval and are transcribed from the same strand (EMBL: AJ550425 and AJ550426, respectively). They also share an identical seed sequence (20). Thus, miRNA221/222 might be generated from the same primary transcript, which could account for the simultaneous enhancement of their expression in tumor cells observed in previous studies $(26,27)$. Importantly, our results show that co-repression of the miR-221/222 cluster compromises cell survival (20). This indicates a functional overlap between miR-221 and miR-222 in controlling cell survival. There are four reports that provide evidence not only on the carcinogenic role of miR-221/222 regulating $\mathrm{p} 27$, but also conferring tamoxifen resistance in breast cancer by targeting p27 (26-29). However, to the best of our knowledge, indirect regulation of miR-221/222 by TFs in the context of cancer cells has not yet been reported.

Using a bioinformatic approach, Shalg et al proposed the concept of miRNA target hubs, that were genes regulated by dozens of miRNA and that were involved in a diversity of developmental processes and in transcription regulation. The miRNA target hubs were highly enriched for developmental processes, specifically for muscle development and nervous system development, as well as for TFs and transcription regulators. A potential regulatory model of the gene expression network is that genes belonging to the same regulon will be coregulated, not only at the transcriptional level, but also posttranscriptionally (23). We think that our results, which identify STAT1 and STAT2 as indirect targets for miR$221 / 222$ in the context of glioma cell lines, demonstrated another regulatory model. Based on our results, after repression of miR-221/222, eight direct mRNA targets of TFs were upregulated (released from the mRNA-3' UTR binding effect), then the TFs interacted with other TFs (such as STAT1 and STAT2), finally regulating gene expression. The miRNA-TF-TF-genes model of miR-221/222-TFs-STAT1/2gene expression has uncovered another anti-cancer mechanism via the repression of miR-221/222 in U251 glioma cells. It is well known that miRNAs function to fine tune gene expression through their target mRNAs; however, the changes in the expression levels of the eight mRNAs remain undetectable in our study.

Type I IFNs (IFN- $\alpha$ and IFN- $\beta$ ) bind to a common receptor and are crucial effector cytokines in many antiviral, antiproliferative, and immune responses $(30,31)$. Binding of IFN $\alpha / \beta$ to the type I receptor results in transphosphorylation and activation of the Jak1/Tyk 2 kinase $(32,33)$. Type I IFN receptor (IFNAR) activation triggers the JAK-mediated tyrosine phosphorylation of STAT2 and STAT1 (34). The activated kinases elicit tyrosine phosphorylation and dimerization of STAT1 and STAT2, which subsequently form a heterotrimeric transcription factor complex with ISGF-3, and activate genes that contain an ISRE (interferon-stimulated response element) site to modulate the transcription of over 300 genes (35). The interferon-induced protein with tetratricopeptide repeats (IFIT) 1, IFIT2, IFIT3, and IFIT5 were upregulated 4.22 - to 39.48 -fold, as indicated by the microarray results.

We also observed upregulation of STAT1, STAT2 and ISGF3- $\gamma$ subunit expression after repression of the miR$221 / 222$ cluster in U251 glioma cells. STAT1 is a key factor not only in signal transduction coupled to IFNAR, but also coupled to the type II IFN (i.e., IFN- $\gamma$ ) receptor (36). In addition, activation of STAT1 can occur in response to other cytokines, such as IL-6 (37). STAT1 promotes apoptosis and negatively regulates cell growth, metastasis and angiogenesis (38-41). STAT1 is deficient or inactive in many types of human tumors, although some tumors have activated STAT1 (38-40). The reconstitution of STAT1 suppressed the tumorigenicity of RAD-105 cells in vivo, which correlated with decreased expression of proangiogenic molecules, such as bFGF, MMP-2 and MMP-9 (41). Consequently, STAT1 might act as an important inhibitor of tumor angiogenesis and therefore negatively regulates tumor growth and metastasis, and tumor-intrinsic STAT1 is an important mediator for antiangiogenic signals, such as IFN (41). By contrast, STAT2 is implicated in only IFNAR-coupled signal transduction (36).

In conclusion, our results suggest that repression of the miR-221/222 cluster suppresses the growth and progression of glioma, at least in part by upregulating STAT1 and STAT2 mRNA translation and activating their function. These findings provide a mechanistic rationale for the overexpression miR-221/222 cluster in glial carcinogenesis. Additional functional studies are now required to understand the molecular basis of the formation of glioma, and to allow the development of innovatively tailored therapies targeting specific tumor markers, and that act as vaccine adjuvants for cancer therapy.

\section{Acknowledgements}

Grant support: Supported by China National Natural Scientific Found (30901772, 30772231, to C.S. Kang and C.Z. Zhang, respectively), and Program for New Century Excellent Talents in University (NCET-07-0615).

\section{References}

1. Lee RC and Ambros V: An extensive class of small RNAs in Caenorhabditis elegans. Science 294: 862-864, 2001.

2. Calin GA and Croce CM: MicroRNA signatures in human cancers. Nat Rev Cancer 6: 857-866, 2006.

3. Esquela-Kerscher A and Slack FJ: Oncomirs - microRNAs with a role in cancer. Nat Rev Cancer 6: 259-269, 2006.

4. He L and Hannon GJ: MicroRNAs: Small RNAs with a big role in gene regulation. Nat Rev Genet 5: 522-531, 2004.

5. Calin GA, Sevignani C, Dumitru CD, et al: Human microRNA genes are frequently located at fragile sites and genomic regions involved in cancers. Proc Natl Acad Sci USA 101: 2999-3004, 2004

6. Cimmino A, Calin GA, Fabbri M, et al: miR-15 and miR-16 induce apoptosis by targeting BCL2. Proc Natl Acad Sci USA 102: 13944-13949, 2005.

7. Mercatelli N, Coppola V, Bonci D, et al: The inhibition of the highly expressed mir-221 and mir-222 impairs the growth of prostate carcinoma xenografts in mice. PLoS One 3: e4029, 2008. 
8. Cancer Genome Atlas Research Network: Comprehensive genomic characterization defines human glioblastoma genes and core pathways. Nature 455: 1061-1068, 2008.

9. Kim S, Dougherty E, Shmulevich I, et al: Identification of combination gene sets for glioma classification. Mol Cancer Ther 1: 1229-1236, 2002.

10. Rickman DS, Bobek MP, Misek DE, et al: Distinctive molecular profiles of high-grade and low-grade gliomas based on oligonucleotide microarray analysis. Cancer Res 61: 6885-6891, 2001.

11. Fuller GN, Mircean C, Tabus I, et al: Molecular voting for glioma classification reflecting heterogeneity in the continuum of cancer progression. Oncol Rep 14: 651-656, 2005.

12. Shai R, Shi T, Kremen TJ, et al: Gene expression profiling identifies molecular subtypes of gliomas. Oncogene 22: 4918-4923, 2003.

13. Godard S, Getz G, Delorenzi M, et al: Classification of human astrocytic gliomas on the basis of gene expression: a correlated group of genes with angiogenic activity emerges as a strong predictor of subtypes. Cancer Res 63: 6613-6625, 2003.

14. Freije WA, Castro-Vargas FE, Fang Z, et al: Gene expression profiling of gliomas strongly predicts survival. Cancer Res 64 6503-6510, 2004.

15. Ciafre SA, Galardi S, Mangiola A, et al: Extensive modulation of a set of microRNAs in primary glioblastoma. Biochem Biophys Res Commun 334: 1351-1358, 2005.

16. Yang YH, Dudoit S, Luu P, et al: Normalization for cDNA microarray data: A robust composite method addressing single and multiple slide systematic variation. Nucleic Acids Res 30: e15, 2002.

17. Shannon P, Markiel A, Ozier O, et al: Cytoscape: a software environment for integrated models of biomolecular Interaction networks. Genome Res 13: 2498-2504, 2003.

18. Ramkissoon SH, Mainwaring LA, Sloand EM, Young NS and Kajigaya S: Nonisotopic detection of microRNA using digoxigenin labeled RNA probes. Mol Cell Probes 20: 1-4, 2006.

19. Kang CS, Zhang ZY, Jia ZF, et al: Suppression of EGFR expression by antisense or small interference RNA inhibits U251 glioma cell growth in vitro and in vivo. Cancer Gene Ther 13: 530-538, 2006 .

20. Zhang C, Kang C, You Y, et al: Co-suppression of miR-221/ 222 cluster suppresses human glioma cell growth by targeting p27kip1 in vitro and in vivo. Int J Oncol 134: 1653-1660, 2009

21. Guo Y, Guo H, Zhang L, et al: Genomic analysis of antihepatitis $B$ virus (HBV) activity by small interfering RNA and lamivudine in stable HBV-producing cells. J Virol 79: 14292-14303, 2005.

22. MAQC Consortium, Shi L, Reid LH, et al: The microarray quality control (MAQC) project shows inter- and intraplatform reproducibility of gene expression measurements. Nat Biotechnol 24: 1151-1161, 2006.

23. Shalgi R, Lieber D, Oren M and Pilpel Y: Global and local architecture of the mammalian microRNA-transcription factor regulatory network. PLoS Comput Biol 3: e131, 2007.

24. Takamizawa J, Konishi H, Yanagisawa K, et al: Reduced expression of the let-7 microRNAs in human lung cancers in association with shortened postoperative survival. Cancer Res 64: 3753-3756, 2004.
25. Iorio MV, Ferracin M, Liu CG, et al: MicroRNA gene expression deregulation in human breast cancer. Cancer Res 65: 7065-7070, 2005.

26. Le Sage C, Nagel R, Egan DA,et al: Regulation of the p27(Kip1) tumor suppressor by miR-221 and miR-222 promotes cancer cell proliferation. EMBO J 26: 3699-3708, 2007.

27. Visone R, Russo L, Pallante P, et al: MicroRNAs (miR)-221 and miR-222, both overexpressed in human thyroid papillary carcinomas, regulate p27Kip1 protein levels and cell cycle. Endocr Relat Cancer 14: 791-798, 2007.

28. Galardi S, Mercatelli N, Giorda E, et al: miR-221 and miR-222 expression affects the proliferation potential of human prostate carcinoma cell lines by targeting p27Kip1. J Biol Chem 282: 23716-23724, 2007.

29. Miller TE, Ghoshal K, Ramaswamy B, et al: MicroRNA$221 / 222$ confers tamoxifen resistance in breast cancer by targeting p27(Kip1). J Biol Chem 283: 29897-29903, 2008.

30. Ferrantini M, Capone I and Belardelli F: Interferon-alpha and cancer: mechanisms of action and new perspectives of clinical use. Biochimie 89: 884-893, 2007.

31. Belardelli F and Gresser I: The neglected role of type 1 interferon in the $\mathrm{T}$-cell response: implications for its clinical use. Immunol Today 17: 369-372, 1996.

32. Kisseleva T, Bhattacharya S, Braunstein J and Schindler CW: Signaling through the JAK/STAT pathway, recent advances and future challenges. Gene 285: 1-24, 2002.

33. Jove R: Preface: STAT signaling. Oncogene 19: 2466-2467, 2000.

34. Bluyssen HA and Levy DE: Stat2 is a transcriptional activator that requires sequence-specific contacts provided by STAT1 and p48 for stable interaction with DNA. J Biol Chem 272: 4600-4605, 1997.

35. Dale TC, Rosen JM, Guille MJ, Lewin AR, Porter AG, Kerr IM and Stark GR: Overlapping sites for constitutive and induced DNA binding factors involved in interferon-stimulated transcription. EMBO J 8: 831-839, 1989.

36. Stark GR, Kerr IM, Williams BRG, Silverman RH and Schreiber RD: How cells respond to interferons. Annu Rev Biochem 67: 227-264, 1998.

37. Schindler C and Strehlow I: Cytokines and STAT signaling. Adv Pharmacol 47: 113-174, 2000.

38. Clifford JL, Walch E, Yang X, et al: Suppression of type I interferon signaling proteins is an early event in squamous skin carcinogenesis. Clin Cancer Res 8: 2067-2072, 2002.

39. Landolfo S, Guarini A, Riera L, et al: Chronic myeloid leukemia cells resistant to interferon-alpha lack STAT1 expression. Hematol J 1: 7-14, 2000.

40. Wong LH, Krauer KG, Hatzinisiriou I, et al: Interferon-resistant human melanoma cells are deficient in ISGF3 components, STAT1, STAT2, and p48-ISGF3gamma. J Biol Chem 272: 28779-28785, 1997.

41. Huang S, Bucana CD, Van Arsdall M and Fidler IJ: STAT1 negatively regulates angiogenesis, tumorigenicity and metastasis of tumor cells. Oncogene 21: 2504-2512, 2002. 